\title{
Adult Orbital Lesions in Saudi Arabia: A Multi-centered Demographic Study with Clinicopathological Correlation
}

\author{
Abrar K. Alsalamah ${ }^{1}$, Azza MY. Maktabi ${ }^{2}$, Hind M. Alkatan ${ }^{3,4,}$, (D) \\ ${ }^{1}$ Vitreoretinal and Uveitis Divisions, King Khaled Eye Specialist Hospital, Riyadh, Saudi Arabia \\ ${ }^{2}$ Pathology and Laboratory Medicine Department, King Khaled Eye Specialist Hospital, Riyadh, Saudi Arabia \\ ${ }^{3}$ Ophthalmology Department, King Saud University, Riyadh, Saudi Arabia \\ ${ }^{4}$ Pathology Department, King Saud University, Riyadh, Saudi Arabia
}

\section{ARTICLE INFO \\ Article History \\ Received 05 April 2020 \\ Accepted 11 July 2020 \\ Keywords \\ Orbit \\ demographics \\ histopathology \\ metastatic \\ secondary \\ space-occupying lesion \\ tumors}

\begin{abstract}
The demographics, clinical features, and histopathological classification of orbital space-occupying lesions in adults have not been widely described in our part of the world except for the pediatric population. In this retrospective study, we collected 110 consecutive adult patients (18 years and older) with orbital lesions (excluding lacrimal gland lesions) that were diagnosed histopathologically in two tertiary eye centers in Riyadh, Saudi Arabia (January 2000 to July 2017). Patients with thyroid-related orbitopathy, infectious, and inflammatory/pseudo-inflammatory lesions were excluded. We had 60 males (54.5\%) and 50 females (45.5\%). The mean age at presentation was 51.4 years (range 19-99). Proptosis was the most common clinical presentation (mean duration 15.4 months). The orbital lesions in order of increasing prevalence were: lymphoproliferative lesions in $26.4 \%$; vascular in $21.8 \%$; secondary tumors in $14.6 \%$; neurogenic in $13.6 \%$; structural in $10.0 \%$; soft tissue tumors $8.2 \%$; then metastatic tumors $(2.7 \%)$ and others (extramedullary leukemia, fibrous dysplasia, and histiocytic lesion: Rosai-Dorfman disease): one case each. Gender distribution was varied in lymphoproliferative disorders compared to vascular lesions. Cavernous hemangioma was the most common vascular lesion (83.3\%) and schwannoma was the most common neurogenic tumor (60\%). Secondary lesions extended to the orbit mostly from eyelids in nine out of 16 or conjunctiva in four out of 16 cases. A favorable outcome was observed in about $80 \%$ of patients who underwent excisional biopsy. The rest encountered local recurrence of the tumors, growing of residual lesions, and recurrence with further invasion to nearby structures. We concluded having a similar demographic pattern of orbital lesions in adults as has been universally reported. We have fewer secondary tumors. We have summarized the pathological profile of adult orbital lesions according to patients' age, gender, symptoms, and location of the lesion as a baseline guide for proper diagnosis of any orbital mass prior to surgical management planning and for future prognostic studies.
\end{abstract}

(C) 2020 The Authors. Published by Atlantis Press International B.V.

This is an open access article distributed under the CC BY-NC 4.0 license (http://creativecommons.org/licenses/by-nc/4.0/).

\section{INTRODUCTION}

Different types of neoplastic and non-neoplastic lesions can affect the orbit. Orbital lesions can be classified according to the site of origin into primary, secondary, and metastatic. Primary orbital tumors are those that originate from tissues of the orbit. Secondary orbital tumors are defined as neoplasms that have reached the orbit by direct extension from adjacent structures such as the eyelids, conjunctiva, intraocular tissues, paranasal sinuses, nasopharynx, and cranial cavity. Metastatic tumors are those that originate from distant organs [1]. Shields et al. [2] have proposed a thorough classification of orbital space-occupying lesions, in which orbital lesions were categorized into cystic, vasculogenic, peripheral nerve, optic nerve and meningeal, fibrocytic, osseous and fibro-osseous, cartilaginous, lipocytic and myxoid, myogenic, lacrimal gland, primary melanocytic, metastatic, lymphoid and leukemia, secondary, histiocytic, thyroid-related orbitopathy, other inflammatory, and miscellaneous. This classification is based on the tissue type which allows an

"Corresponding author.Email: hindkatan@yahoo.com; hkatan@ksu.edu.sa Data availability statement: The authors confirm that the data supporting the findings of this study are available within the article [and/or] its supplementary materials. organized thinking and easy categorization to the ophthalmologist and the pathologist. A modified classification (Appendix 1) has been used in the present study due to lesser number of cases studied and the lack of cases in some of the above categories, which has been followed by other group of authors in one of the centers and it allows easier comparison from one published series to another [3].

The reported prevalence of the different types of orbital spaceoccupying lesions varies greatly in the literature. For example, the prevalence differs in series derived solely from histopathologically confirmed lesions compared to those derived from clinical diagnosis without histopathological verification (lesions such as thyroid-related orbitopathy, inflammatory pseudo-tumor, capillary hemangioma, and optic nerve tumors, which are less likely to be subjected to surgical excision) [4]. Another variation is due to the age range and geographic area of the study population. Lymphoid, secondary, and metastatic tumors increase significantly with age, whereas cystic orbital lesions decrease with age, thus being the most common in the pediatric age group compared to adults [3]. Similarly, the most common vascular lesion in children was found to be capillary hemangioma while in adults, the main vasculogenic lesion was cavernous hemangioma [5]. 
In this study, we included adult patients aged 18 years and older who underwent a biopsy for a non-lacrimal gland orbital mass (excluding thyroid-related orbitopathy, traumatic, infectious, and inflammatory/pseudo-inflammatory lesions). This study will allow the physician to anticipate the type of orbital lesion based on the age, gender, symptoms, signs, duration, location of the lesion, and other characteristics. Thus, the physician will have baseline data in this part of the world to be able to provide more focused diagnostic and management plan for any patient with an orbital lesion.

\section{PATIENTS AND METHODS}

A retrospective review of adult patients (18 years and older) with orbital lesions who presented to King Khaled Eye Specialist Hospital (KKESH) and King Abdulaziz University Hospital (KAUH) in Riyadh, Saudi Arabia, from January 2000 through July 2017 and had a histopathologically confirmed tissue diagnosis. The study was approved by the Human Ethics Committee/Institutional Review Board at KKESH with an expedited approval on April 18, 2017 as a retrospective study and an approved collaborative agreement with KAUH (RP 1711-R). A general informed consent was obtained from all patients as part of the common practice at both centers. Our inclusion criteria were to include all adult patients who were coded with a diagnosis of an orbital mass or simulating condition for further detailed analysis. Saudi and non-Saudi patients were included. Patients with thyroid-related orbitopathy, traumatic, infectious, inflammatory/pseudo-inflammatory, and lacrimal gland lesions were excluded. Charts were reviewed for demographic data, main presenting symptoms, duration of symptoms, laterality, affected anatomical part of the orbit, presence of systemic illness, and family history of malignancy. Initial visual acuity (VA), intraocular pressure (IOP), presence of proptosis, whether a palpable mass was present or not, presence or absence of tenderness, ocular motility exam, pupil reaction, slit-lamp examination, presence of other findings, type of biopsy (excisional or incisional), patient outcome, and follow-up duration, were retrieved from the medical records using a specially designed data collection sheet. Histopathological slides were retrieved and reviewed to confirm the diagnosis and complete the necessary data for all patients. Immunohistochemical stains were also reviewed to support the confirmed tissue diagnosis. Using a modification of a previously published classification of orbital tumors [2], the final diagnosis in each case was classified into one of the following main categories of lesions: lymphoproliferative, vascular, secondary, neurogenic, structural, soft tissue, metastatic, and others. The number and percentage of the diagnosis of specific lesions within each main category were calculated. Data were analysed using SPSS $^{\circledR}$ version 22.0 (IBM Inc., Chicago, IL, USA). Descriptive analysis was primarily done, where categorical variables were presented in the form of frequencies and percentages and continuous variables in the form of mean \pm Standard Deviation (SD) and range (minimum to maximum).

\section{RESULTS}

We identified 110 adult patients with orbital space-occupying lesions and histopathologically verified tissue diagnoses who were seen, managed, and followed up during the period encompassed by this study (17 years). The total subjects included in this study were
60 males (54.5\%) and 50 females (45.5\%). The age ranged from 19 to 99 years, with a mean age at presentation of 51.4 years (SD of 18.7). A total of 96 patients (87.3\%) were Saudi and $14(12.7 \%)$ were non-Saudi. The final histopathological diagnosis in each case was classified into one of the major categories of lesions: lymphoproliferative, vascular, secondary, neurogenic, structural, soft tissue tumors, metastatic, and others. Lymphoproliferative lesions were found in $29 / 110$ patients (26.4\%), being the most common orbital lesions in our series, followed by vascular lesions in 24/110 (21.8\%), secondary lesions in 16/110 (14.6\%), neurogenic lesions in $15 / 110$ (13.6\%), structural lesions in 11/110 (10\%), soft tissue tumors in nine out of $110(8.2 \%)$, metastatic tumors to the orbit in three of out $110(2.7 \%)$, and others in three out of $110(2.7 \%)$ patients including extramedullary leukemia, fibrous dysplasia, and histiocytic lesion (Rosai-Dorfman disease) in one case each. The histopathological classification of the 110 orbital lesions is shown in Table 1.

Table 1 The histopathological classification of 110 adult patients with orbital lesions

\begin{tabular}{|c|c|}
\hline Category and subcategory & Number of patients (\%) \\
\hline Lymphoproliferative & $29(26.4)$ \\
\hline MZBCL & $6(20.7)$ \\
\hline DLBCL & $5(17.2)$ \\
\hline BRLH & $5(17.2)$ \\
\hline Lymphoma, NOS & $5(17.2)$ \\
\hline Follicular lymphoma & $3(10.3)$ \\
\hline Low grade B-cell lymphoma & $2(6.9)$ \\
\hline Lymphoplasmacytic lymphoma & $1(3.5)$ \\
\hline Mantle cell lymphoma & $1(3.5)$ \\
\hline T-cell lymphoma & $1(3.5)$ \\
\hline Vascular & $24(21.8)$ \\
\hline Cavernous hemangioma & $20(83.3)$ \\
\hline AVM & $2(8.3)$ \\
\hline Lymphangioma & $1(4.2)$ \\
\hline Vascularized bony tumor & $1(4.2)$ \\
\hline Secondary/origin & $16(14.6)$ \\
\hline SCC of eyelid & $5(31.3)$ \\
\hline SCC of conjunctiva & $4(25.0)$ \\
\hline SGC of eyelid & $4(25.0)$ \\
\hline Nasopharyngeal carcinoma & $2(12.5)$ \\
\hline SCC of lacrimal sac & $1(6.3)$ \\
\hline Neurogenic & $15(13.6)$ \\
\hline Schwannoma & $9(60.0)$ \\
\hline Solitary neurofibroma & $4(26.7)$ \\
\hline Orbital meningioma & $2(13.3)$ \\
\hline Structural & $11(10.0)$ \\
\hline Dermoid cyst & $5(45.5)$ \\
\hline Hematic cyst & $5(45.5)$ \\
\hline Mucocele & $1(9.1)$ \\
\hline Soft tissue tumors & $9(8.2)$ \\
\hline SFT & $4(44.5)$ \\
\hline Leiomyosarcoma & $2(22.2)$ \\
\hline Liposarcoma & $2(22.2)$ \\
\hline RB-related sarcoma (MFH) & $1(11.1)$ \\
\hline Metastatic & $3(2.7)$ \\
\hline Breast carcinoma & $2(66.7)$ \\
\hline Cutaneous melanoma & $1(33.3)$ \\
\hline Others & $3(2.7)$ \\
\hline
\end{tabular}

MZBCL, Marginal zone B-cell lymphoma; DLBCL, Diffuse large B-cell lymphoma; BRLH, benign reactive lymphoid hyperplasia; NOS, not otherwise specified; AVM, Arteriovenous malformation; SCC, squamous cell carcinoma; SGC, sebaceous gland carcinoma; SFT, solitary fibrous tumor; $\mathrm{Rb}$, retinoblastoma; $\mathrm{MFH}$, malignant fibrous histiocytoma. 
The distribution of age and gender per each histopathological category is shown in Table 2.

Clinically, the affected eye was the left eye in 54 patients $(49.1 \%)$, while the right eye was affected in 53 patients (48.2\%). Bilateral involvement was seen in three cases (2.7\%): extra-nodal Marginal-zone

Table 2 Demographics of 110 consecutive adult patients per each histopathological category

\begin{tabular}{|c|c|c|c|}
\hline \multirow{2}{*}{ Category } & Age (years) & \multirow{2}{*}{$\begin{array}{c}\text { Male, } \\
n=60 \\
(\%)\end{array}$} & \multirow{2}{*}{$\begin{array}{c}\text { Female, } \\
\begin{array}{c}n=50 \\
(\%)\end{array}\end{array}$} \\
\hline & Mean \pm SD [range $]$ & & \\
\hline Others $(n=3)$ & $37.7 \pm 24.9[19-66]$ & $2(66.7)$ & $1(33.3)$ \\
\hline Soft tissue tumors $(n=9)$ & $39.2 \pm 13.7[22-64]$ & $7(77.8)$ & $2(22.2)$ \\
\hline Structural $(n=11)$ & $39.4 \pm 17.9[19-69]$ & $5(45.5)$ & $6(54.5)$ \\
\hline Neurogenic $(n=15)$ & $41.8 \pm 14.1[21-75]$ & $8(53.3)$ & $7(46.7)$ \\
\hline Vascular $(n=24)$ & $47.5 \pm 16.8[27-87]$ & $8(33.3)$ & $16(66.7)$ \\
\hline Metastatic $(n=3)$ & $48.3 \pm 2.5[46-51]$ & $1(33.3)$ & $2(66.7)$ \\
\hline Lymphoproliferative $(n=29)$ & $60.0 \pm 15.3[27-96]$ & $21(72.4)$ & $8(27.6)$ \\
\hline Secondary $(n=16)$ & $72.6 \pm 15.3[43-99]$ & $8(50.0)$ & $8(50.0)$ \\
\hline
\end{tabular}

B-cell Lymphoma (MZBCL), metastatic breast carcinoma, and RosaiDorfman disease: one case each. Proptosis was the most common encountered presenting symptom in $65(59.1 \%)$ patients followed by decreased vision in $28(25.5 \%)$ patients, ptosis or lid edema, and similarly visible or palpable mass in $27(24.5 \%)$ patients. Other less common clinical complaints were: periocular pain or tenderness (15.5\%), diplopia (7.3\%), globe displacement (5.5\%), and ocular motility limitation $(2.7 \%)$. The frequency of the presenting complaints for each histopathological category and the mean duration in months for each symptom are depicted in Figures 1 and 2. Most of our patients were free of any medical illnesses (62.4\%) prior to the presentation. Family history of malignancy was absent in all patients.

The mean visual acuity at initial presentation was 0.5 LogMAR (Logarithm of the Minimum Angle of Resolution). Intraocular pressure measurement was in the range of $10-35 \mathrm{mmHg}$ (mean 17.3, SD of 4.7). Proptosis was clinically evident in 71 patients $(64.5 \%)$ followed by a palpable mass which was felt in 38 patients (34.5\%) and described as mobile in $12(31.6 \%)$, diffuse in configuration in 19 (50\%), and circumscribed in 15 (39.5\%). The details of the rest of the clinical findings are summarized in Table 3.

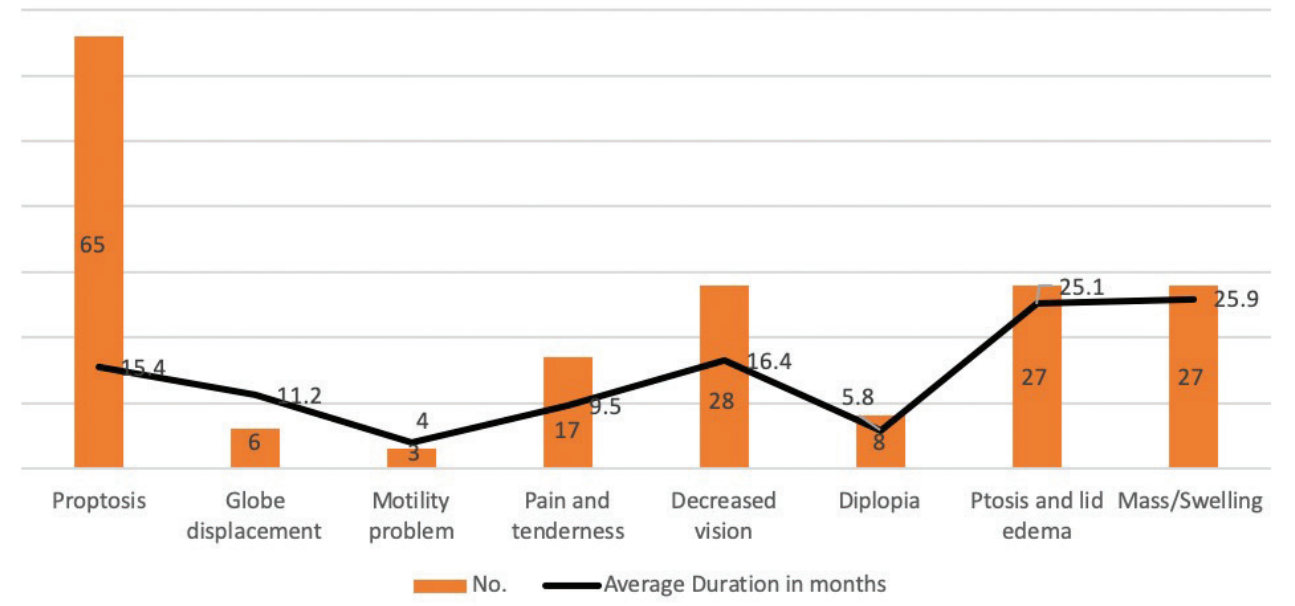

Figure $1 \mid$ Frequency of the presenting complaints and the mean duration in months of 110 consecutive adult patients with orbital lesions.

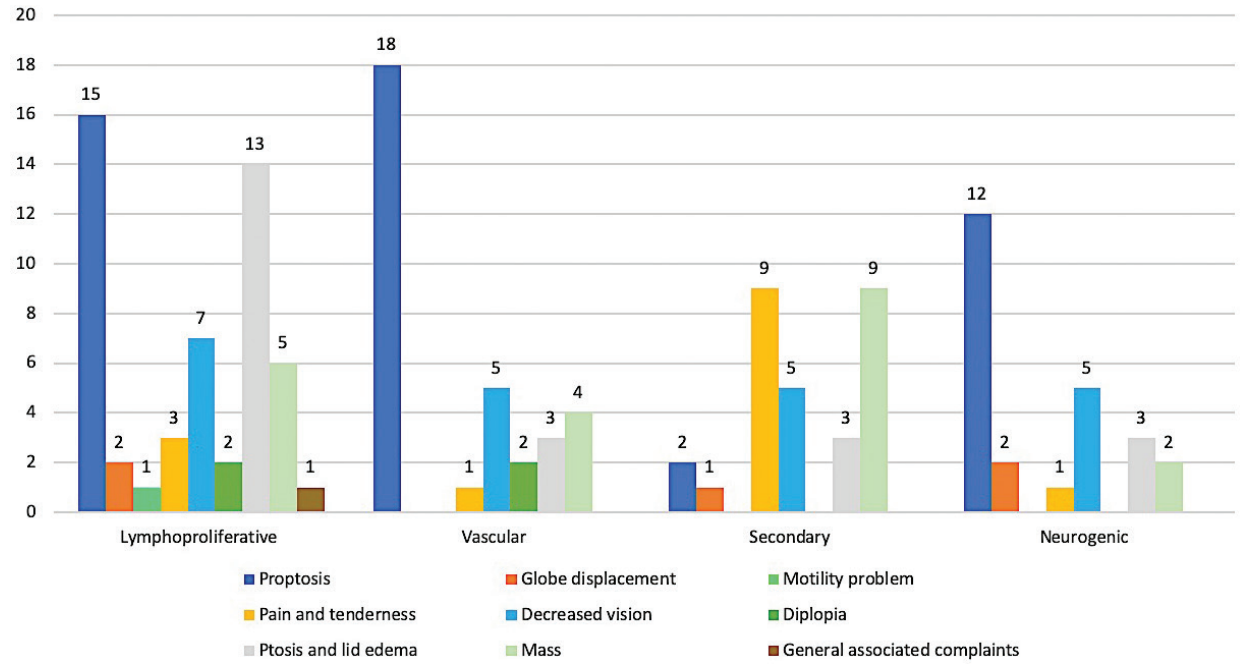

Figure 2 Distribution of the clinical symptoms per each histopathological category. 
The location of the orbital lesions was categorized based on the affected anatomical part of the orbit into three main categories: anterior, posterior (extraconal, intraconal, and combined extraconal and intraconal), and periocular. Lymphoproliferative lesions were found that it mostly affect the anterior orbit in 17/29 (58.6\%) patients, and equally in the posterior orbit and periocular area in six $(20.7 \%)$ patients each. Vascular lesions were mostly identified in the posterior orbit (70.8\%), followed by anterior orbit $(25 \%)$, and secondary lesions were mostly observed in the periocular area or anterior orbit. Neurogenic and structural lesions were mostly found posteriorly in $73.3 \%$, and $54.5 \%$, respectively. Soft tissue tumors' location was variable depending on the tumor type. The location of all lesions is demonstrated in Table 4.

Table 3 Clinical characteristics of 110 consecutive adult patients with orbital lesions

\begin{tabular}{lc}
\hline Clinical characteristics & Number of patients (\%) \\
\hline VA LogMAR, mean \pm SD [range], median & $0.5 \pm 0.8[0-3], 0.2$ \\
IOP mmHg, mean \pm SD [range], median & $17.3 \pm 4.7[10-35], 16$ \\
Proptosis & $71(64.5)$ \\
Palpable mass & $38(34.5)$ \\
Mobility & $12(31.6)$ \\
Configuration & \\
$\quad$ Circumscribed & $15(39.5)$ \\
Diffuse & $19(50.0)$ \\
$\quad$ Not specified & $4(10.5)$ \\
Tenderness & $12(10.9)$ \\
Pupil reaction & \\
Reactive & $89(80.9)$ \\
Non-reactive & $7(6.4)$ \\
Sluggish & $6(5.5)$ \\
Not specified & $8(7.3)$ \\
Ocular motility & \\
Full & $64(58.2)$ \\
Limited & $46(41.8)$ \\
Optic disc & \\
Normal & $86(78.2)$ \\
Swollen & $9(8.2)$ \\
Pale & $7(6.4)$ \\
Not specified & $8(7.3)$ \\
Fundus & \\
Normal & $89(80.9)$ \\
Irrelevant other fundus findings & $11(10.0)$ \\
Choroidal folds & $2(1.8)$ \\
Not specified & $8(7.3)$ \\
\hline &
\end{tabular}

Excisional biopsy was performed in $63(57.3 \%)$ patients, while $47(42.7 \%)$ patients had initial incisional biopsy for further nonsurgical treatment. The mean follow-up duration was 9.6 months with a range from 1 to 244 months. Among 63 patients who had an excisional biopsy, 50 (79.4\%) patients had a good outcome with no recurrence, nor further growth of their lesions. Ten patients had unfavorable outcomes either due to local recurrence only following incomplete surgical excision or treatment for lymphoma in eight $(12.7 \%)$, or recurrence with further invasion or extension of their tumors in two (3.2\%) patients. The remaining three $(4.8 \%)$ patients had regrowth of residual incompletely excised primary lesions described during surgery as being adherent to adjacent nerves and/or muscles upon further follow up (Figure 3). The specific diagnoses for the different outcomes are listed in Table 5.

\section{DISCUSSION}

In this study, we conducted a retrospective review of the clinical records and the histopathological reports of adult patients (18 years and older) with orbital lesions who had confirmed tissue diagnosis over the course of 17 years. Previous reported series of orbital space-occupying lesions in adults showed variable results, this could be related to different factors such as the nature and geographic location of the reporting center and source of the material

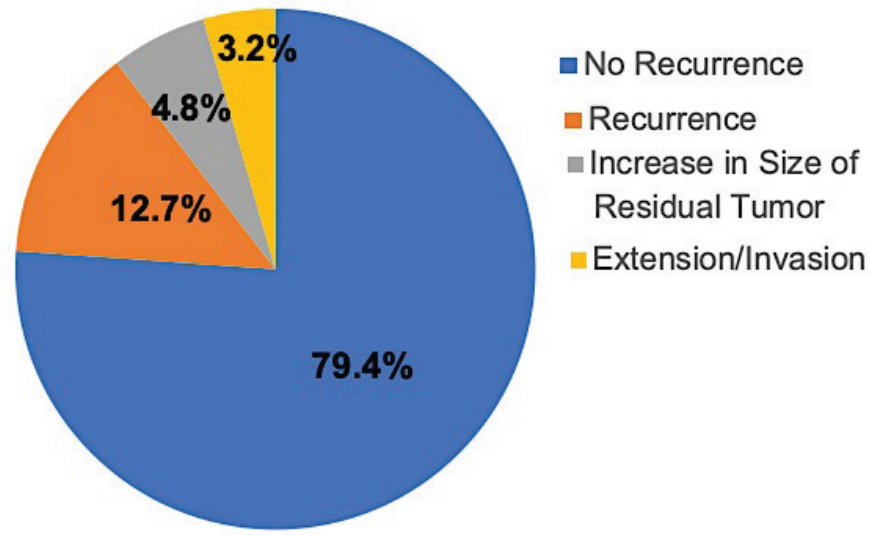

Figure 3 Distribution of the outcomes of 63 orbital lesions post-excisional biopsy.

Table 4 Affected anatomical part of the orbit per each histopathological category

\begin{tabular}{|c|c|c|c|c|c|}
\hline \multirow[b]{2}{*}{ Category } & \multirow{2}{*}{$\begin{array}{c}\text { Anterior } \\
\text { orbit } \\
(n=43)\end{array}$} & \multicolumn{3}{|c|}{ Posterior orbit $(n=47)$} & \multirow{2}{*}{$\begin{array}{c}\text { Periocular } \\
(n=20)\end{array}$} \\
\hline & & $\begin{array}{l}\text { Extraconal } \\
\qquad(n=15)\end{array}$ & $\begin{array}{l}\text { Intraconal } \\
\quad(n=27)\end{array}$ & $\begin{array}{c}\text { Intraconal and } \\
\text { extraconal }(n=5)\end{array}$ & \\
\hline Lymphoproliferative $(n=29)$ & 17 & 3 & 3 & 0 & 6 \\
\hline Vascular $(n=24)$ & 6 & 4 & 12 & 1 & 1 \\
\hline Secondary $(n=16)$ & 7 & 0 & 1 & 0 & 8 \\
\hline Neurogenic $(n=15)$ & 4 & 3 & 8 & 0 & 0 \\
\hline Structural $(n=11)$ & 3 & 3 & 2 & 1 & 2 \\
\hline Soft tissue tumors $(n=9)$ & 4 & 1 & 0 & 1 & 3 \\
\hline Metastatic $(n=3)$ & 1 & 0 & 1 & 1 & 0 \\
\hline Others $(n=3)$ & 1 & 1 & 0 & 1 & 0 \\
\hline
\end{tabular}


Table 5 Summary of the outcome in 13 cases post-excisional biopsy/chemotherapy in correlation with the final diagnosis

\begin{tabular}{|c|c|c|c|c|c|c|}
\hline Category & $\begin{array}{l}\text { Recurrence } \\
\quad(n=8)\end{array}$ & Diagnosis & $\begin{array}{l}\text { Regrowth of } \\
\text { residual tumor } \\
\text { (incomplete } \\
\text { excision) } \\
(n=3)\end{array}$ & Diagnosis & $\begin{array}{c}\text { Extension/ } \\
\text { invasion } \\
(n=2)\end{array}$ & Diagnosis \\
\hline Lymphoproliferative $(n=29)$ & 3 & $\begin{array}{c}\text { Two Follicular lymphoma, } \\
\text { one BRLH }\end{array}$ & 0 & & 0 & \\
\hline $\operatorname{Vascular}(n=24)$ & 0 & & $1^{* *}$ & Cavernous hemangioma & 0 & \\
\hline Secondary $(n=16)$ & 3 & $\begin{array}{c}\text { Two SCC of conjunctiva, } \\
\text { one SGC of eyelid }\end{array}$ & 0 & & 2 & $\begin{array}{c}\mathrm{SCC}^{+} \text {of conjunctiva } \\
\mathrm{SGC}^{++} \text {of eyelid }\end{array}$ \\
\hline Neurogenic $(n=15)$ & 1 & Schwannoma & $1^{* *}$ & $\begin{array}{c}\text { Schwannoma } \\
\text { Solitary neurofibroma }\end{array}$ & 0 & \\
\hline Structural $(n=11)$ & 0 & & 0 & & 0 & \\
\hline Soft tissue tumors $(n=9)$ & 1 & Liposarcoma $^{+++}$ & 0 & & 0 & \\
\hline Metastatic $(n=3)$ & 0 & & 0 & & 0 & \\
\hline Others $(n=3)$ & 0 & & 0 & & 0 & \\
\hline
\end{tabular}

reviewed. Bonavolontà et al. [6] analyzed different orbital lesions of 2480 patients presenting during a period of 35 years in Italy and found that the most frequent orbital lesion was dermoid cyst in 339 (14\%) followed by non-Hodgkin lymphoma in 291 (12\%) and cavernous hemangioma in 218 (9\%). Since we have only included adult patients, we had fewer structural lesions with only five dermoid cysts. However, the rest of our findings were well aligned with the above, where lymphoproliferative lesions were the most common orbital lesions in $29(26.4 \%)$ cases followed by vascular lesions in $24(21.8 \%)$ cases, most of which were cavernous hemangiomas. Demirci et al. [7] also found a high prevalence of lymphoid tumors accounting for $28 \%$ but his review involved patients aged 60 years or older. Shinder et al. [8] reviewed 268 consecutive patients with orbital masses over a 10 -year period and found that secondary orbital tumors (26\%) and lymphoproliferative lesions (25\%) were the most common orbital lesions in their series. Similar results were also reported by Johansen et al. [9] in his review of 965 biopsied orbital lesions during a 24-year period in which secondary tumors $(20.9 \%)$ were the most common followed by lymphoid tumors (13.4\%). We had relatively lesser secondary tumors $(14.6 \%)$ possibly reflecting proper management and adequate complete excisions of primary tumors involving adjacent structures such as eyelids, sinuses or nasopharynx. Ohtsuka et al. [10] studied orbital tumors in Japanese patients over a period of 20 years and found that among the 244 cases, $213(89 \%)$ were primary orbital tumors and the most common tumors in the primary group were malignant lymphoma (27.7\%) and reactive lymphoid hyperplasia (21.1\%). Shields et al. [4] however, in their retrospective case series of 1264 consecutive patients referred for a suspected orbital tumor or tumor-simulating condition during a 30-year period reported that vascular lesions were the most common (17\%) followed by secondary orbital tumors $(11 \%)$ and lymphoid or leukemic lesions accounted for only $10 \%$. This variation might be due to the fact that only cases with tissue diagnosis have been enrolled in our study.
Among our lymphoproliferative lesions, extra-nodal MZBCL was the most common in $20.7 \%$ followed by diffuse large B-cell lymphoma in $17.2 \%$. This is somehow similar to the previous report in Italy [6].

Regarding vascular lesions, Khan et al. [11] found that cavernous hemangioma was the most common vascular lesion in their series of 81 vascular lesions accounting for $59.25 \%$. Similarly, in the two studies mentioned earlier by Demirci and Shields [4,7], cavernous hemangioma was again the most common among the vasculogenic lesions in $54 \%$ and $36 \%$, respectively. Our study has shown the highest prevalence of cavernous hemangioma in $83.3 \%$ of all vascular lesions and these were mostly intraconal in location. The prevalence of orbital venous malformation has been found by Bonavolontà et al. [12] to be increasing over a period of 40 years (1977-2017). The authors recommended better diagnostic modalities and accurate management plan to avoid unnecessary surgical intervention that might carry risk of complications [12].

Secondary orbital tumors were the third most frequent orbital lesions in our series, with lesions from squamous cell carcinoma of either the eyelid or conjunctiva in nine out of 16 cases thus being the most common underlying primary tumor in this category followed by sebaceous gland carcinoma of the eyelid in four out of $16(25 \%)$ cases. Similar findings were reported by Khan et al. [11] who found that among the secondary orbital lesions in his series, lesions of the eyelids were the most common in $62.50 \%$ and among those eyelid lesions, squamous cell carcinoma was seen in more than half (61.38\%) followed by basal cell carcinoma (11.72\%) and sebaceous gland carcinoma (8.28\%). Shields et al. [4], on the other hand, found that the most common origin for their secondary orbital tumors was intraocular in 54 cases, followed by eyelid origin in 25 cases, and conjunctival origin in 22 cases. Malik and El Sheikh studied 854 lesions involving the eye and adnexae in Sudanese patients and found that nasopharyngeal carcinoma was the most common origin for secondary orbital tumors in $30.9 \%$ of all secondary 
lesions compared to two of 16 (12.5\%) cases only in our series [13]. Interestingly, we did not encounter any case of basal cell carcinoma extending to the orbit, while Gunalp and Gunduz in their review of 524 patients with secondary orbital tumors found that basal cell carcinoma was the most common primary eyelid lesion in 129 (24.6\%) cases [14].

The most common neurogenic lesions in our series were schwannoma in nine out of $15(60 \%)$ cases and solitary neurofibroma in four out of $15(26.7 \%)$ cases. It is important to note that patients with plexiform neurofibroma were not included in our series, as most of them did not need a biopsy as they were known to have type 1 neurofibromatosis. Similar results were also reported by Shields et al. [4] who found that among 23 patients with peripheral nerve lesions, $14(61 \%)$ were schwannomas and six (26\%) were neurofibromas.

In our series, the most common structural lesions were dermoid cysts-despite the fact that it was conducted among adults-and hematic cysts accounting for five out of 11 cases each. We would expect to have more structural lesions if lacrimal gland dacryops (as structural lesions) were included in our study. Seregard and Sahlin [15] reviewed 300 patients with space-occupying orbital lesions being managed over a period of 24 years and found that dermoid cyst was the most common structural lesion in 27 out of 37 patients in this group.

Solitary Fibrous Tumors (SFTs) were the most common soft tissue tumors in our series (44.5\%). The number of orbital SFTs reported in the literature has been increasing especially with the use of immunohistochemical techniques [16].

The metastatic tumors to the orbit in our series were from breast carcinoma in two out of three cases and cutaneous melanoma in one case. Shields and Bonavolontà $[6,17]$ concluded in their two studies that the most primary tumor site for metastatic orbital lesions was the breast in 53\% and 39\%, respectively. Having metastatic orbital lesions with an unknown primary has also been reported [18]. Interestingly, Char et al. [18] in his study of metastatic orbital lesions has shown an initial 11/31 patients (35\%) without a known primary malignancy. Even following radiological studies and proper investigations, the primary malignancy was not diagnosed in eight out of these cases accounting for $26 \%$ of the total patients.

Demographics in our study have shown a general male predominance $(54.5 \%)$ compared to females (45.5\%), which is almost identical to what was reported by Roh et al. [19] who showed a gender distribution of orbital tumors of 52\% males and $48 \%$ females. This male predilection was even more evident among patients with lymphoproliferative lesions in our study where males accounted for $72.4 \%$ and the male $(\mathrm{M})$ to female $(\mathrm{F})$ ratio was 2.6:1. Male predilection among patients who had lymphoproliferative lesions with $61 \%$ male involvement was also reported in a study in Iran [20]. Vascular lesions, on the other hand, were more common in females (66.7\%) (F:M ratio of 2:1). In a retrospective analysis of 66 cases with the diagnosis of cavernous hemangioma, Harris and Jakobiec [21] also found a F:M ratio of approximately 7:4. The mean age at presentation in our series was 51.4 years with a range of [19.0-99.0] which is relatively similar to what has been reported by Shields et al. (mean age of 45 years [range 0-92]) [4]. However, we have observed that the mean age - when analyzed based on the diagnosis of different tumor categories - was variable since lymphoproliferative and secondary lesions had tendency to affect patients who are 60 years or older (mean age of 60 and 72.6 years, respectively). The mean age was $<60$ years in the remaining categories (including the metastatic lesions) as demonstrated clearly in Table 2.

The clinical manifestations also varied widely among different categories depending on the most prevalent location as mentioned earlier and the tumor behavior. However, proptosis was the most frequent presenting symptom in our series, followed by ptosis, periorbital mass, and decreased vision. In patients with lymphoproliferative disease, proptosis followed by ptosis and lid edema were the prominent features reflecting the variable location of involvement in this category. Proptosis was the most prominent feature in vascular and neurogenic lesions. On the other hand, pain, tenderness and a palpable mass were more encountered with secondary orbital tumors possibly due to the associated invasive behavior and extension. Similar findings were reported by Demirci et al. [7] who found that the most common clinical features at presentation were a periorbital palpable mass (26\%), followed by proptosis (18\%) and pain (15\%). Markowski et al. [22] found that the most frequent manifestations were proptosis in $100 \%$ of patients, eyelid swelling in $54 \%$, limitation of eye movement in $45 \%$, decreased visual acuity in $43 \%$, pain in $30 \%$, and diplopia in $16 \%$. In our series, we observed that most of the lymphoproliferative lesions were in the anterior orbit (extraconal) while the majority of the vascular and neurogenic lesions arose intraconally. Comparable results were reported by Shinder et al. [8] in his review who found that 63 out of 68 lymphoproliferative lesions were located extraconally while 13 out of 20 vascular lesions were found in the intraconal space.

We did not intend to study the detailed follow up, outcome and the prognosis in our study; however, we observed a relatively good outcome in almost $80 \%$ of our cases that underwent excisional biopsy in an attempt for complete excision of the orbital lesions. The unfavorable outcomes were related to true recurrence with or without further invasion or extension. We have also encountered two recurrent cases of follicular lymphoma years following excision and management by the oncology team. These might not reflect the true rate of recurrence among lymphoproliferative patients, since these were usually diagnosed by incisional biopsy then referred for further non-surgical management in a general tertiary hospital. Three cases also presented with regrowth of residual tumors because of difficult complete excision during the primary surgery (where free margins were difficult to be assured). Recurrence has been reported variably among different orbital lesions reaching up to $25.7 \%$ in lacrimal gland tumors and $21.3 \%$ in vasogenic lesions followed by neurogenic tumors (recurrence in 16.6\%), secondary tumors (9.5\%), and lympho-hematopoietic tumors in $2.8 \%$ of cases [23]. Our cases were demonstrated as an observation but were not studied further since this was not within the scope of our study.

\section{CONCLUSION}

In summary, this study was undertaken to determine the demographics and clinicopathological correlation of various orbital lesions in adults in Saudi Arabia since we have already identified this in the pediatric age group in a previously published paper. The most common adult orbital lesions in order of frequency were lymphoproliferative lesions (26.4\%); vascular (21.8\%); secondary orbital tumors (14.6\%); neurogenic lesions (13.6\%); structural 
lesions (10\%); soft tissue tumors (8.2\%); and metastatic tumors to the orbit $(2.7 \%)$. This distribution is generally similar to what has been reported in the USA, Italy, and Japan. Patient's age must be taken into consideration when evaluating a patient with an orbital mass since secondary tumors, lymphoproliferative disorders, and metastatic lesions tend to occur more in older adults (with a mean age of $72.6,60$, and 48.3 years, respectively). Gender variation is also another observation. Clinically, proptosis was the most common symptom but other clinical features were variable and had correlated well with the type of lesion and its most frequent location. Residual re-growing orbital lesions, and recurrences with or without further invasion were major causes of disease-related morbidity. Therefore, we recommend further national studies in the future for the assessment of management modalities and detailed outcomes with prognostic analysis based on the basic information provided in our study.

\section{CONFLICTS OF INTEREST}

The authors declare they have no conflicts of interest.

\section{AUTHORS' CONTRIBUTION}

AKA and AMM contributed in data collection, entry, analysis and writing manuscript (original draft). HMA contributed in study conceptualization and writing (review, editing, and preparation for submission) the manuscript.

\section{FUNDING}

No funds were received from any of the institutions.

\section{REFERENCES}

[1] Costin BR, Perry JD, Foster JA. Classification of orbital tumors. In: Perry JD. Clinical ophthalmic oncology. Berlin, Heidelberg: Springer-Verlag; 2014, pp. 9-14.

[2] Shields JA, Bakewell B, Augsburger JJ, Flanagan JC. Classification and incidence of space-occupying lesions of the orbit: a survey of 645 biopsies. Arch Ophthalmol 1984;102;1606-11.

[3] Alkatan HM, Al Marek F, Elkhamary S. Demographics of pediatric orbital lesions: a tertiary eye center experience in Saudi Arabia. J Epidemiol Glob Health 2019;9;3-10.

[4] Shields JA, Shields CL, Scartozzi R. Survey of 1264 patients with orbital tumors and simulating lesions: the 2002 Montgomery Lecture, part 1. Ophthalmology 2004;111;997-1008.

[5] Shields JA, Bakewell B, Augsburger JJ, Donoso LA, Bernardino V. Space-occupying orbital masses in children: a review of 250 consecutive biopsies. Ophthalmology 1986;93;379-84.
[6] Bonavolontà G, Strianese D, Grassi P, Comune C, Tranfa F, Uccello G, et al. An analysis of 2,480 space-occupying lesions of the orbit from 1976 to 2011. Ophthal Plas Receonstr Surg 2013;29;79-86.

[7] Demirci H, Shields CL, Shields JA, Honavar SG, Mercado GJ, Tovilla JC. Orbital tumors in the older adult population. Ophthalmology 2002;109;243-8.

[8] Shinder R, Al-Zubidi N, Esmaeli B. Survey of orbital tumors at a comprehensive cancer center in the United States. Head Neck 2011;33;610-14.

[9] Johansen S, Heegaard S, Bøgeskov L, Prause JU. Orbital spaceoccupying lesions in Denmark 1974-1997. Acta Ophthalmol Scand 2000;78;547-52.

[10] Ohtsuka K, Hashimoto M, Suzuki Y. A review of 244 orbital tumors in Japanese patients during a 21-year period: origins and locations. Jpn J Ophthalmol 2005;49;49-55.

[11] Khan AA, Sarwar S, Sadiq MAA, Ur Rehman M, Ullah A, Ahmad I. Analysis of 1246 cases of orbital lesions: a study of 17 years. Nat Sci 2015;7;324-37.

[12] Bonavolontà $\mathrm{P}$, Fossataro F, Attanasi F, Clemente L, Iuliano A, Bonavolontà G. Epidemiological analysis of venous malformation of the orbit. J Craniofac Surg 2020;31;759-61.

[13] Malik MOA, El Sheikh EH. Tumors of the eye and adnexa in the Sudan. Cancer 1979;44;293-303.

[14] Günalp I, Gündüz K. Secondary orbital tumors. Ophthal Plast Reconstr Surg 1997;13;31-5.

[15] Seregard S, Sahlin S. Panorama of orbital space-occupying lesions. The 24-year experience of a referral centre. Acta Ophthalmol Scand 1999;77;91-8.

[16] Bernardini FP, de Conciliis C, Schneider S, Kersten RC, Kulwin DR. Solitary fibrous tumor of the orbit: is it rare? Report of a case series and review of the literature. Ophthalmology 2003;110;1442-8.

[17] Shields JA, Shields CL, Brotman HK, Carvalho C, Perez N, Eagle RC. Cancer metastatic to the orbit: the 2000 Robert M. Curts Lecture. Ophthal Plast Reconstr Surg 2001;17;346-54.

[18] Char DH, Miller T, Kroll S. Orbital metastases: diagnosis and course. Br J Ophthalmol 1997;81;386-90.

[19] Roh KK, Lee JH, Youn DH. Clinical analysis of tumors of the eye and its adnexa. Korean J Ophthalmol 1988;2;27-31.

[20] Asadi-Amoli F, Nozarian Z, Bonaki HN, Mehrtash V, Entezari S. Clinicopathologic assessment of ocular adnexal lymphoproliferative lesions at a tertiary eye hospital in Iran. Asian Pac J Cancer Prev 2016;17;3727-31.

[21] Harris GJ, Jakobiec FA. Cavernous hemangioma of the orbit. J Neurosurg 1979;51;219-28.

[22] Markowski J, Jagosz-Kandziora E, Likus W, Pająk J, MrukwaKominek E, Paluch J, et al. Primary orbital tumors: a review of 122 cases during a 23-year period: a histo-clinical study in material from the ENT Department of the Medical University of Silesia. Med Sci Monit 2014;20;988-94.

[23] Tang W, Hei Y, Xiao L. Recurrent orbital space-occupying lesions: a clinicopathologic study of 253 cases. Chin J Cancer Res 2013;25;423-9. 


\section{APPENDIX}

Appendix $1 \mid$ Shields modified classification of orbital lesions

Cystic:

- Dermoid cyst

- Mucocele

- Epithelial cyst

- Microphthalmos with cyst

- Lacrimal gland cyst

- Hematic cyst

Inflammatory simulating tumor:

- Inflammatory pseudotumor

- Myositis

- Sarcoid nodule

Adipose containing:

- Dermolipoma

- Liposarcoma

Vasculogenic:

- Capillary hemangioma

- Cavernous hemangioma

- Orbital varix

- Lymphangioma

- Arteriovenous malformation

Rhabdomyosarcoma

Secondary/metastatic:

- Neuroblastoma

- Retinoblastoma with orbital extension

Lacrimal gland/fossa:

- Dacryoadenitis

- Ectopic lacrimal gland

- Epithelial tumors ${ }^{*}$

- Dacryops ${ }^{*}$

Lymphoid tumor/leukemia:

- Acute lymphatic leukemia

- Burkitt's lymphoma

- Granulocytic (Myeloid) sarcoma

Optic nerve/meningeal:

- Optic nerve glioma

- Orbital meningioma

Peripheral nerve tumor:

- Neurofibroma

Osseous, fibro-osseous, cartilaginous:

- Osteoma

- Fibrous histiocytoma

- Fibro sarcoma

- Fibrous dysplasia

- Proliferative nodular fasciitis

- Solitary fibrous tumor

Histiocytic:

- Infantile xanthogranuloma

- Histiocytosis $\mathrm{x}$

Melanocytic

Miscellaneous:

- Pilomatrixoma

- Malignant small cell tumor

"Proposed by Alkatan et al. [3]. "Previously classified as Hemangiopericytoma (Vasculogenic). 